\title{
Ce dont notre avenir pourra être fait
}

\section{Jean Martin}

Dr méd., membre de la rédaction

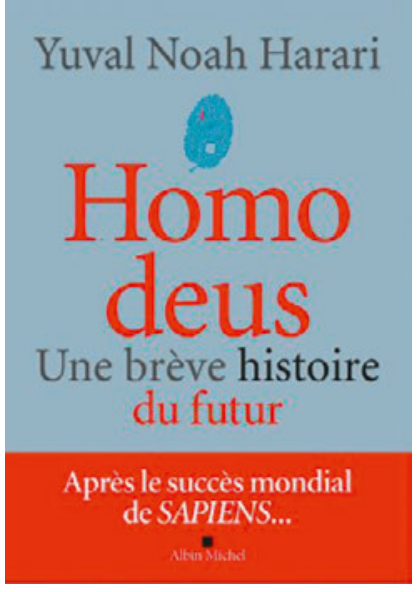

Yuval Noah Harari

Homo deus

Une brève histoire du futur Paris: Albin Michel; 2017. 463 pages. $39.80 \mathrm{CHF}$. ISBN 978-2226393876

Yuval Noah Harari (1976) est ce professeur d'université israélien dont un premier livre, Sapiens - Une brève histoire de l'humanité, a connu un succès international depuis 2015. Il publie un nouveau pavé sur l'histoire de l'avenir! Expliquant (il n'est pas le seul!) que plus rien ne sera comme avant. «Toutes les prédictions qui parsèment ce livre ne sont qu'une tentative pour aborder les dilemmes d'aujourd'hui et une invitation à changer le cours de l'avenir.»

\section{Avant, avoir le pouvoir voulait dire accéder aux données. Aujourd'hui c'est savoir ce qu'il faut ignorer.}

Homo deus a trois parties: 1) Homo sapiens conquiert le monde (y compris: quelle différence entre les humains et les autres animaux); 2) Homo sapiens donne sens au monde (ou comment l'humanisme est devenu la plus importante des religions); 3) Homo sapiens perd le contrôle (en quoi biotechnologies et intelligence artificielle menacent l'humanisme, en promouvant une nouvelle élite de surhommes dominateurs).
Ce qui suit ne saurait résumer un livre aussi multidimensionnel, plein d'informations de sources diverses, surprenant, parfois métaphorique. Avec de nombreuses références à des évènements scientifiques et historiques grands ou petits (y compris l'étonnant cheval Hans le Malin, en Allemagne vers 1900, et le chimpanzé d'un zoo suédois qui sait prévoir/planifier). Alliant à la science dure des apports de philosophie, psychologie et sociologie.

\section{Pour planter le décor}

L'Homme a-t-il achevé son évolution? «Il n'y a aucune raison de penser que Sapiens soit le dernier stade. Qui sait ce qui pourrait résulter de changements de notre $\mathrm{ADN}$ ou de la structure de notre cerveau [...] Le génie biologique ne va pas attendre que la sélection naturelle opère. Les bio-ingénieurs vont réécrire délibérément le code génétique, recâbler les circuits cérébraux. Le génie cyborg fusionnera le corps organique avec des appareils comme des mains bioniques, des yeux artificiels ou des nanorobots.» On cherchera aussi à développer des êtres entièrement non organiques - en remplaçant par exemple les réseaux neuronaux par un logiciel intelligent.

"Au XXI ${ }^{\mathrm{e}}$ siècle, la frontière entre l'histoire et la biologie est susceptible de se brouiller, parce que des fictions idéologiques serviront à réécrire des brins d'ADN, des intérêts politiques et économiques réaménageront le climat. La réalité intersubjective avalera la réalité objective.»

\section{Poussées, vers l'immortalité, le bonheur, et être comme des dieux}

Dans l'introduction: «Avec les niveaux de prospérité atteints, l'humanité se fixera probablement comme prochains objectifs l'immortalité, le bonheur et la divinité. Nous allons chercher à triompher de la vieillesse et de la mort; après avoir tiré les gens de la misère, nous voudrons les rendre vraiment heureux, et hisser les hommes au rang des dieux» - par des potentialités que jusqu'ici nous attachons à ces derniers. Ces avancées ne profiteront pas à tous mais à une classe privilégiée: «La messe est dite: l'égalité est hors jeu.» 
«Les rêves d'immortalité et de divinité ne laissent pas de déconcerter. Malgré l'hubris technologique qui les caractérise, idéologiquement ce n'est pas nouveau. Voici trois cents ans que le monde est dominé par l'humanisme, qui sanctifie la vie et le pouvoir d'Homo sapiens. On ne va que pousser ces idéaux humanistes à leur conclusion logique.»

\section{Heurs et malheurs de l'humanisme et de la démocratie}

L'humanisme qu'a connu l'histoire moderne sera remplacé par autre chose, dit Harari. Il décrit trois grandes branches de l'humanisme jusqu'ici: le libéral, le socialiste (y compris la tentative communiste) et l'évolutionniste, dont un exemple est le modèle nazi (surprenant, allez au texte pour en savoir plus - pp. 270 à 280). Il traite surtout de l'humanisme libéral. «L'antidote à une existence vide de sens et de lois nous a été fourni par l'humanisme au cours des derniers siècles. Alors que traditionnellement le plan cosmique donnait du sens à la vie, l'humanisme renverse les rôles et attend des expériences intérieures des humains qu'elles donnent sens à tout l'univers.»

\section{L'intelligence sera obligatoire, la conscience optionnelle.}

«Elargir nos horizons peut cependant se retourner contre nous en semant la confusion et en nous rendant plus passifs qu'avant [...] Dans le passé, la censure opérait en bloquant l'afflux de l'information. Au XXI' siècle, elle opère en inondant la population d'informations non pertinentes. Avant, avoir le pouvoir voulait dire accéder aux données. Aujourd'hui cela veut dire savoir ce qu'il faut ignorer.»

«Il est envisageable que les technologies du XXI ${ }^{\mathrm{e}}$ siècle dépouillent les hommes de leur autorité pour en investir des algorithmes non humains.» Menaces sur la démocratie: «Qu'adviendra-t-il le jour où nous comprendrons que les choix des clients et des électeurs ne sont jamais libres et où nous disposerons de technologies pour calculer, concevoir ou dépasser leurs sentiments?» "Avec le changement des conditions du traitement des données, la démocratie pourrait décliner et même disparaître. Des institutions vénérables comme les élections et les parlements peuvent devenir obsolètes: non qu'elles soient contraires à l'éthique mais parce qu'elles ne sauraient traiter les données assez efficacement [...] La tortue gouvernementale ne saurait rattraper le lièvre technologique.»

\section{Science, religion, histoire(s)}

Harari parle beaucoup de ces thèmes. «Il est courant de représenter l'histoire de la modernité comme un combat entre science et religion. En théorie, toutes deux s'intéressent par dessus tout à la vérité. En fait, ni l'une ni l'autre ne s'en soucient vraiment: elles peuvent donc aisément trouver des compromis, co-exister et même coopérer [...] En tant qu'institutions collectives, science et religion font passer l'ordre et le pouvoir avant la vérité. Elles sont donc de bons compagnons de route.»

A l'avenir, «la croyance à des mythes nationaux et religieux pourrait déboucher sur une guerre où des millions de gens perdraient leur foyer, leurs membres, voire la vie. La cause de la guerre est fictionnelle, mais la souffrance est réelle à 100\% [...] La fiction n'est pas mauvaise. Sans histoires communément acceptées, aucune société humaine complexe ne saurait fonctionner. Mais ces histoires ne doivent pas devenir nos buts ni nos étalons.» «Grâce à la bio-ingéniérie, la différence entre fiction et réalité se brouillera, tandis que les gens remodèleront la réalité au gré de leurs fictions favorites.»

\section{Pouvoir et sens dans la modernité}

«La modernité est un deal, dont nous sommes tous partie prenante dès notre naissance. Le contrat peut se résumer en une phrase: les hommes acceptent d'abandonner le sens en échange du pouvoir. Jusque dans les Temps modernes, la plupart des cultures attribuaient aux humains un rôle dans le dessein cosmique. Ce plan était l'œuvre des dieux, les humains étaient comme des acteurs sur scène, le script limitait strictement leur jeu [...] En échange de ce renoncement au pouvoir, les prémodernes croyaient que leur vie gagnait du sens. Qu'ils se battent vaillamment sur le camp de bataille,

\section{Le problème de la rareté des ressources pour-} rait être résolu, mais la némésis de l'économie est l'effondrement écologique.

soutiennent le roi [...] avait une réelle importance. S'il arrivait quelque chose de terrible (guerre, épidémie, famine), ils se consolaient: nous ne sommes pas dans le secret du scénario mais pouvons être assurés que tout ce qui arrive a une fin, un but. La culture moderne rejette cette croyance en un grand plan cosmique. La vie n'a ni scénario, ni dramaturge, l'univers est un processus aveugle et sans dessein.» 


\section{La technologie nous remplace(ra) dans beaucoup de tâches - conséquences?}

"Qu'adviendra-t-il du marché du travail dès lors que l'intelligence artificielle surpassera les humains? Qu'adviendra-t-il des relations, des familles et des fonds de pension quand les nanotechnologies et la médecine régénérative feront des octogénaires les nouveaux cinquantenaires?» «L'aubaine technologique permettra de nourrir les masses inutiles. Mais qu'est-ce qui pourra

\section{Bientôt (par Kindle), les livres vous liront pendant que vous les lisez.}

les tenir occupées et les satisfaire? Les gens ont besoin de faire quelque chose, sous peine de devenir fous. La drogue ou les jeux vidéo pourraient être une des réponses - les inutiles passeront toujours plus de temps dans la réalité virtuelle en $3 \mathrm{D}$, qui leur procurera plus d'intensité émotionnelle que la glauque réalité extérieure.»

«Même si vous vous préoccupez plus de justice que de victoire, vous choisirez probablement de remplacer vos soldats et pilotes par des robots et drones autonomes. Les soldats massacrent, violent, pillent. Des ordinateurs programmés selon des algorithmes éthiques se conformeraient bien plus aisément aux derniers arrêts de la Cour pénale internationale»... "Quelqu'un pour donner un coup de frein? Personne ne sait où sont les freins. Par ailleurs, si nous parvenions tant bien que mal à freiner, notre économie s'effondrera en même temps que notre société.»

\section{La question des (masses de) données, le dataïsme}

Le chapitre 11 traite du dataïsme, la religion des data. «Tandis que le système global de traitement des données devient omniscient et tout puissant, la connexion au système devient la source de tout sens.» «Peut-être arriverons-nous à un stade où il sera impossible de se déconnecter, ne serait-ce qu'un instant, de ce réseau omniscient, où la déconnexion signifiera la mort.» «Des appareils comme le Kindle d'Amazon sont capables de recueillir des données sur leurs utilisateurs.
Un Kindle perfectionné saura ce qui vous a fait rire, rendu triste ou mis en colère. Bientôt les livres vous liront pendant que vous les lisez. Et alors que vous aurez vite fait d'en oublier la majeure partie, Amazon lui n'oubliera jamais rien!» $\mathrm{Au} \mathrm{XXI}^{\mathrm{e}}$ siècle, nos données personnelles seront probablement la ressource la plus précieuse que les humains puissent encore offrir, et nous les donnons aux géants de la technologie en échange de services de messagerie et de vidéos de chats.

\section{Perspectives?}

Sur l'évolution éthique du monde: «Les hommes sont menacés de perdre leur valeur économique parce que l'intelligence est découplée de la conscience. L'intelligence est [sera] obligatoire, la conscience optionnelle.» «Epidémies et sécheresses n'ont pas de sens mais nous pouvons les éradiquer. Les guerres ne sont pas un mal nécessaire et nous pouvons faire la paix. Nul paradis ne nous attend après la mort mais nous pouvons créer le paradis ici, sur terre.» Pourtant: «La toute-puissance est là, presque à notre portée, mais sous nos pas s'ouvre l'abysse béant du néant complet; aucune autre culture n'a été davantage en proie à une angoisse existentielle.»

\section{Quelqu'un pour donner un coup de frein? Personne ne sait où sont les freins.}

«Il est dangereux de confier notre avenir aux forces du marché, parce que ces forces font ce qui est bon pour le marché et non ce qui est bon pour l'humanité. La main du marché est aveugle aussi bien qu'invisible; elle pourrait bien rester passive devant la menace du réchauffement climatique ou les dangers de l'intelligence artificielle.» "Nous avons des chances de triompher du problème de la rareté des ressources. [Mais] la véritable némésis de l'économie moderne est l'effondrement écologique. Le progrès et la croissance prennent place dans une biosphère fragile, leurs ondes de choc déstabilisent le milieu [...] Nous devons aussi nous inquiéter qu'une apocalypse écologique aura[it] des conséquences différentes en fonction des castes humaines. Il n'y pas de justice dans l'histoire. Les pauvres souffrent toujours bien plus.» 